\title{
Structural Dynamics of Synapses in Vivo Correlate with Functional Changes during Experience-Dependent Plasticity in Visual Cortex
}

\author{
Daniela Tropea, ${ }^{1 \star}$ Ania K. Majewska, ${ }^{2 \star}$ Rodrigo Garcia, ${ }^{1}$ and Mriganka Sur ${ }^{1}$ \\ ${ }^{1}$ Department of Brain and Cognitive Sciences, Picower Institute for Learning and Memory, Massachusetts Institute of Technology, Cambridge, \\ Massachusetts 02139, and 2Department of Neurobiology and Anatomy, Center for Visual Science, University of Rochester, Rochester, New York 14642
}

The impact of activity on neuronal circuitry is complex, involving both functional and structural changes whose interaction is largely unknown. We have used optical imaging of mouse visual cortex responses and two-photon imaging of superficial layer spines on layer 5 neurons to monitor network function and synaptic structural dynamics in the mouse visual cortex in vivo. Total lack of vision due to dark-rearing from birth dampens visual responses and shifts spine dynamics and morphologies toward an immature state. The effects of vision after dark rearing are strongly dependent on the timing of exposure: over a period of days, functional and structural changes are temporally related such that light stabilizes spines while increasing visually driven activity. The effects of long-term light exposure can be partially mimicked by experimentally enhancing inhibitory signaling in the darkness. Brief light exposure, however, results in a rapid, transient, NMDA-dependent increase of cortical responses, accompanied by increased dynamics of dendritic spines. These findings indicate that visual experience induces rapid reorganization of cortical circuitry followed by a period of stabilization, and demonstrate a close relationship between dynamic changes at single synapses and cortical network function.

\section{Introduction}

Although the importance of structural changes for brain development and function has long been recognized (Ramón y Cajal, 1904), understanding structure-function relationships, particularly at individual synapses in the intact brain, has remained elusive. Rapid changes in synaptic function are thought to precede slower consolidating changes in synaptic structure and network connectivity. Recent work challenges this view, showing that dendritic spine structure can remodel rapidly (Mataga et al., 2004; Matsuzaki et al., 2004; Oray et al., 2004; Noguchi et al., 2005; Holtmaat et al., 2006). The contribution of rapid structural remodeling to functional plasticity is not well understood (Rittenhouse and Majewska, 2009).

Pronounced experience-dependent plasticity in visual cortex provides an opportunity to examine the relationship between synaptic structure and network function in particular detail (Majewska and Sur, 2006). In animals reared in darkness from birth, functional changes in visual cortical neurons include reduced spatial acuity, orientation and direction selectivity, along

\footnotetext{
Received March 30, 2010; revised June 8, 2010; accepted June 30, 2010.

This work was funded by grants from the National Institutes of Health [EY007023 and EY017098 (M.S.); 1F32EY017240 (D.T.); EY019277 (A.K.M.)]. A.K.M. was funded by the Burroughs-Wellcome Career Award in biological sciences, an Arthur P. Sloan Fellowship, and a grant from the Whitehall Foundation. We thank Hongbo Yu for help and useful discussions and Cassandra Lamantia for experimental assistance.

${ }^{*}$ D.T. and A.K.M. contributed equally to this work.

Correspondence should be addressed to Ania K. Majewska, Department of Neurobiology and Anatomy, University of Rochester Medical School, 601 Elmwood Ave., Box 603, Rochester, NY 14642. E-mail: Ania_Majewska@ urmc.rochester.edu.

D. Tropea's present address: Trinity College Dublin, St James Hospital, Dublin 8, Ireland.

DOI:10.1523/JNEUROSCI.1661-10.2010

Copyright $\odot 2010$ the authors $\quad$ 0270-6474/10/3011086-10\$15.00/0
}

with reduced cortical responsiveness (Czepita et al., 1994; Fagiolini et al., 1994). This is accompanied by structural changes in dendritic spine morphology (Winkelmann et al., 1976; Wallace and Bear, 2004), as well as molecular changes that hint at both altered presynaptic (Yang et al., 2002) and postsynaptic (Cotrufo et al., 2003; Tropea et al., 2006) function, and changes in intracellular and extracellular signaling (Lander et al., 1997; Tropea et al., 2006). Exposure to light following dark-rearing reverses many of these effects, restoring molecular (Philpot et al., 2001; Tropea et al., 2001; Cotrufo et al., 2003), structural (Valverde, 1971; Wallace and Bear, 2004) and functional (Buisseret et al., 1982; Li et al., 2006) properties of cortical neurons. While some of these changes can be quite rapid, occurring within minutes (Brakeman et al., 1997) to hours (Buisseret et al., 1982; Philpot et al., 2001; Cotrufo et al., 2003) following light exposure, the timescales, progression and mechanisms of how dark-rearing and subsequent light exposure affects synapses and visual cortical networks are unclear.

To examine whether and how the structure of single synapses relates to the function of cortical networks, we used two forms of in vivo imaging - intrinsic signal optical imaging of functional responses to visual stimulation, and two-photon imaging of the structural dynamics of dendritic spines on layer 5 pyramidal neurons - in the mouse visual cortex. We mapped the progression of change in control and dark-reared mice, as well as in mice that were exposed to light following dark-rearing. Over long timescales (days) of light exposure, we found a gradual increase in cortical responsiveness with a concomitant decrease in spine motility, demonstrating a close inverse relationship between network function and structural spine dynamics. Over rapid timescales (hours) of light exposure, however, there was a dramatic 
disconnection between synapse structure and cortical function: cortical responses increased while dendritic protrusions became highly motile and were rapidly formed and subsequently eliminated. Our experiments demonstrate that visual experience leads to coordinated changes in synapse structure and function within intact cortical circuits, and reveal transient mismatches following perturbations before structure-function relationships are progressively reestablished.

\section{Materials and Methods}

Animals. Male and female mice were reared in a normal light dark cycle (12 h light/12 h light) or in the dark since birth, and injections of anesthetic were performed in the darkness with the aid of night vision goggles before imaging. Mice were all imaged at $28 \pm 1 \mathrm{~d}$ after birth, at the peak of the "critical period" (Gordon and Stryker, 1996), therefore animals reexposed to light for 2 and $7 \mathrm{~d}$ were placed in regular rearing conditions starting from P26 and P21, respectively. In specific experiments, CPP (Sigma; $10 \mathrm{mg} / \mathrm{kg}$ body weight, i.p.) (Frenkel and Bear, 2004) was administered $30 \mathrm{~min}$ before animals were exposed to light. In other experiments, Diazepam (Sigma; in saline with $1 \%$ DMSO; $10 \mathrm{mg} / \mathrm{kg}$ body weight, i.p.) was injected daily in the darkness with night vision goggles for $7 \mathrm{~d}$ before the imaging session. All experiments were performed under protocols approved by the Institutional Animal Care and Use Committee at MIT or University of Rochester and conformed to National Institutes of Health guidelines.

Two-photon imaging. For two-photon imaging, mice (C57BL/6) expressing GFP in a subset of layer 5 cortical neurons (GFP-M; Feng et al., 2000) were used. Mice were anesthetized with avertin (16 $\mu \mathrm{g} / \mathrm{g}$ body weight, i.p.); the skull was exposed, cleaned and glued to a thin metal plate. Primary visual cortex (V1) was identified according to stereological coordinates. The skull above the imaged area was thinned with a dental drill. During surgery and imaging, the animal's temperature was kept constant with a heating pad and the anesthesia was maintained with periodic administration of avertin. Imaging and data analysis were performed as previously described (Majewska et al., 2006). A custom-made two-photon scanning microscope (Majewska et al., 2000) was used, using a wavelength of $920 \mathrm{~nm}$ and a $20 \times 0.95$ numerical aperture objective lens (Olympus) at $10 \times$ digital zoom. Images were acquired as $z$ stacks ( 1 $\mu \mathrm{m}$ step size) every $5 \mathrm{~min}$ for $1-2 \mathrm{~h}$. Analysis was performed with custom macros in Matlab and ImageJ. A small number of images were maxintensity projected in the $z$ plane and each protrusion was analyzed at $400 \%$ digital zoom. Length was measured from the tip of the protrusion to the dendritic shaft. A motility index was calculated for each protrusion based on the average absolute change in length (from the dendrite to the tip of the protrusion) per unit time. For measurement of spine turnover, the area was imaged in the first session and a map of the blood vessels was taken as a reference point. In the following imaging sessions the animal was anesthetized and the skull reexposed. The blood vessels map and dendritic architecture were used to identify the same imaging regions. We concentrated our chronic imaging on two time points- before light exposure and either $2 \mathrm{~h}$ or $2 \mathrm{~d}$ after light exposure, as the extensive skull thinning required to locate GFP-labeled neurons in visual cortex at these ages makes it difficult to carry out more than two imaging sessions. In some cases three imaging sessions were possible and these data are presented without statistical analysis. Dendritic protrusions were identified as persistent if they were located within $0.7 \mu \mathrm{m}$ laterally on the subsequent imaging session. Elimination and formation rates refer to the numbers of new spines and lost spines, respectively, observed on the second imaging time point divided by the total number of spines present in the first imaging session. Survival fractions were computed as the percentage of spines maintained at an imaging time point subsequent to the initial spine population. For two-photon experiments, analysis was performed blind to the experimental manipulation. Classification of spines was based on approximate measurements made on magnified projected images according to the criteria described previously (Harris and Kater, 1994; Oray et al., 2006). The spine classes described are not meant to be absolute and spine measurements indicated a continuum of spine morphologies in the population. However, this classification is a
A

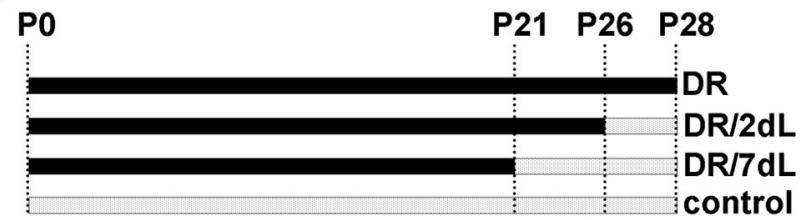

B

DR

DR/2dL
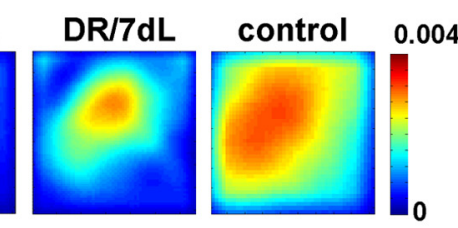

C
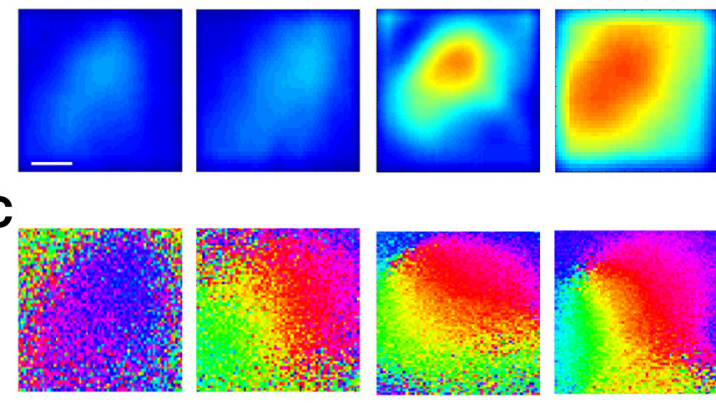

*

\section{D}

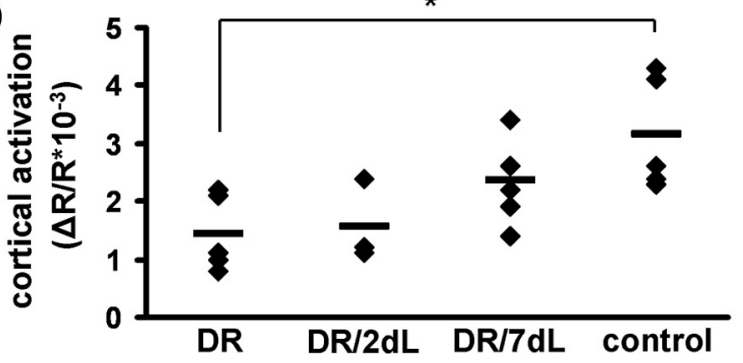

$-36^{\circ}$

Figure 1. Visual experience regulates cortical responses to vision. $\boldsymbol{A}$, Time course of the experiment: all animals were imaged at $P 28$. The period of dark rearing is represented as a black line, while the white-dotted pattern represents the period of exposure to a normal light environment. DR animals were maintained and anesthetized before imaging in complete darkness. DR/2 dl animals were dark-reared until P26 and then exposed to light. DR/7 dl animals were dark-reared until P21 and then exposed to light. Control animals were reared in a normal $12 \mathrm{~h}$ light/ $12 \mathrm{~h}$ dark environment. $\boldsymbol{B}$, Representative images of the cortical intrinsic signal in response to a visual stimulus in individual mice from the different groups imaged. Red hues indicate strong activation, according to color key at right depicting the change in reflectance, $\mathrm{dR} / \mathrm{R}$. In dark-reared mice, cortical activity in response to light is low and increases as mice are exposed to light. Scale bar, $0.5 \mathrm{~mm}$. C, Representative retinotopic maps of visual field elevation in individual mice with different visual experience. The map is highly disorganized in darkreared animals and the level of organization progressively increases together with the duration of light exposure. Color key at right depicts visual elevation. D, Quantification of the visually evoked optical signal across animals with different visual experience. The strength of the signal (normalized change in reflectance, $\mathrm{dR} / \mathrm{R}$ ) is low in dark-reared animals and recovers with longer light exposure. ${ }^{*} p<0.05$ when compared with control.

simple measure of different types of spine morphologies which have been linked to synapse development and plasticity (Matsuzaki et al., 2001, 2004; Oray et al., 2006). In some cases, injections of cholera toxin subunit B (CTB, List Biologic) coupled to Alexa Fluor 594 (Invitrogen) were made adjacent to imaged areas to facilitate identification after fixation. Mice were transcardially perfused and fixed with paraformaldehyde and coronal sections were cut to verify the location of imaged cells using an atlas of the mouse brain (Franklin and Paxinos, 1997).

Intrinsic signal optical imaging. For optical imaging of intrinsic signals, mice $(\mathrm{C} 57 \mathrm{BL} / 6)$ were anesthetized with urethane $(1.5 \mathrm{mg} / \mathrm{kg}$, i.p. $)$, and the skull was thinned as described above. A custom-made attachment was used to fix the head and minimize movement. The cortex was covered with agarose solution (1.5\%) and a glass coverslip. During the imaging session the animal's body temperature was kept constant with a heating blanket and the EKG was continuously monitored. The eyes were periodically treated with silicone oil and the animal was allowed to breathe pure oxygen. Red light $(630 \mathrm{~nm})$ was used to illuminate the cortical surface, and the change of luminance was captured by a CCD 

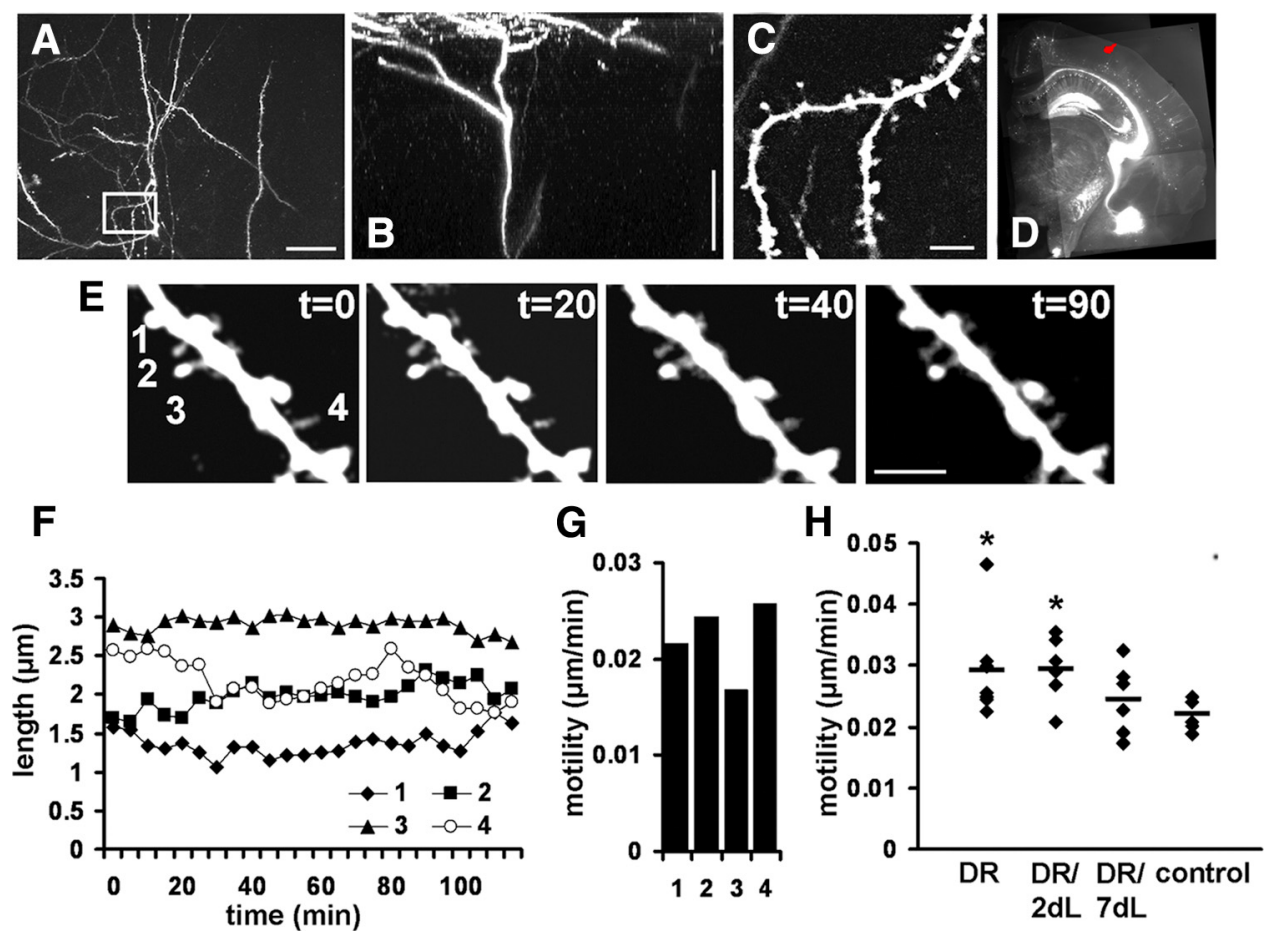

Figure 2. Visual experience regulates dendritic spine structure in the visual cortex. $\boldsymbol{A}$, In vivo two-photon image of the apical tuft of a layer 5 pyramidal neuron in visual cortex of a P28 mouse. The image is a collapsed $z$ stack showing the extent of the apical tuft dendritic arbor. Scale bar, $100 \mu \mathrm{m}$. $\boldsymbol{B}$, Three-dimensional reconstruction showing the dendritic arbor from the side. Scale bar, 100 $\mu \mathrm{m}$. C, Two-photon image of dendritic spines in vivo from dendrite shown in A (boxed area). Scale bar, $5 \mu \mathrm{m}$. D, Following imaging, red tracer was injected into the imaged area based on the blood vessel pattern. The animal was perfused and the imaged area identified in fixed section. The injection site is shown in red and resides in visual cortex as identified using coordinates of the mouse brain atlas. $\boldsymbol{E}$, Time-lapse image of dendritic spines in the visual cortex of a control mouse in vivo. Images shown were taken 30 min apart. Scale bar, $2 \mu \mathrm{m}$. Spines are motile at these ages (notice spine 3 withdraws into the dendrite during the first hour of imaging). $\boldsymbol{F}$, Lengths of the 4 spines at left are shown plotted over $2 \mathrm{~h}$. $\mathbf{G}$, The motility index for the same four spines showing the approximate range of motilities observed in control animals (filopodia are not shown in this figure-filopodia were generally more motile and were rarely observed in control animals). $\boldsymbol{H}$, Spine motility indices for P28 mice exposed to different visual environments. Analysis included all spine classes and filopodia. There is a significant increase in spine motility in visually deprived animals (DR) compared with controls. Two days of exposure to a normal light-dark cycle does not affect motility but after $7 \mathrm{~d}$ of exposure to normal dark-light conditions the motility index is no longer significantly different from that in control animals. ${ }^{*} p<0.05$ compared with control.

camera (Cascade 512B, Roper Scientific) during the presentation of visual stimuli (STIM, Optical Imaging). The screen was placed $20 \mathrm{~cm}$ away from the mouse head and both eyes were simultaneously stimulated. Custom software was developed to control the image acquisition and synchronization between the camera and stimuli. An elongated horizontal or vertical white bar $\left(9^{\circ} \times 72^{\circ}\right)$ over a uniformly gray background was drifted continuously through the up-down dimension of the visual field. After moving to the last position, the bar would jump back to the initial position and start another cycle of movement-thus, the chosen region of visual space $\left(72^{\circ} \times 72^{\circ}\right)$ was stimulated in periodic fashion $(9 \mathrm{~s} / \mathrm{cycle})$. Images of visual cortex were continuously captured at a rate of 15 frames/s during each stimulus session of $25 \mathrm{~min}$. For data analysis, a temporal high pass filter (135 frames) was used to remove slow noise components, after which the temporal Fast Fourier Transform (FFT) component at the stimulus frequency $\left(9 \mathrm{~s}^{-1}\right)$ was calculated pixel by pixel from the whole set of images. No spatial averaging was done. The amplitude of the FFT component was used to measure the strength of visually driven responses.

Statistical analysis. Statistical comparisons used the two-tailed MannWhitney test and compared average values obtained in individual animals. $p<0.05$ was considered significant. All data are presented as mean \pm SEM.

\section{Results}

Visual experience affects the functional activation of visual cortex

The maturation of cortical circuitry is dependent on patterned activity supplied by sensory stimulation (Sherman and Spear, 1982; Li et al., 2006). To determine how vision affects cortical activity and organization in mouse $\mathrm{V} 1$, we used intrinsic signaling optical imaging to examine this area in 4 sets of mice that experienced different visual environments (Fig. $1 A$ ). In the first set, mice were dark-reared from birth until P28 (DR) and never experienced normal vision. Two sets of mice were dark-reared from birth until either P26 (DR/2 dl) or P21 (DR/7 dl) after which they were exposed to a normal $12 \mathrm{~h}$ light/dark cycle until the imaging session (P28). In the last set, mice were reared in a normal $12 \mathrm{~h}$ light-12 h dark cycle from birth until P28 (control). Dark-reared mice $(n=5)$ showed a marked decrease in the amplitude of visually evoked cortical activity when compared with control mice $(n=5)$ reared in a normal light environment $(p<0.01$; Fig. $1 B, D)$. In addition it appeared that dark-reared mice had poorly organized cortical maps of retinotopy (Fig. 1C; supplemental Fig. $\mathrm{S} 1$, available at www.jneurosci.org as supplemental material).

Two days of light exposure following dark rearing resulted in a more ordered cortical map of visual space but no increase in visually driven cortical activity $(n=3 ; p>0.05$ when compared with dark-reared animals). Seven days of light exposure refined the retinotopic map and increased visually evoked cortical activation to control levels $(n=6 ; p>0.05$ when compared with control animals). This is in agreement with previous studies of visual deprivation showing that dark rearing prevents the maturation of cortical circuits and response properties (Fagiolini et al., 1994), and that subsequent light exposure can induce normal cortical development (Buisseret et al., 1982; Li et al., 2006). 

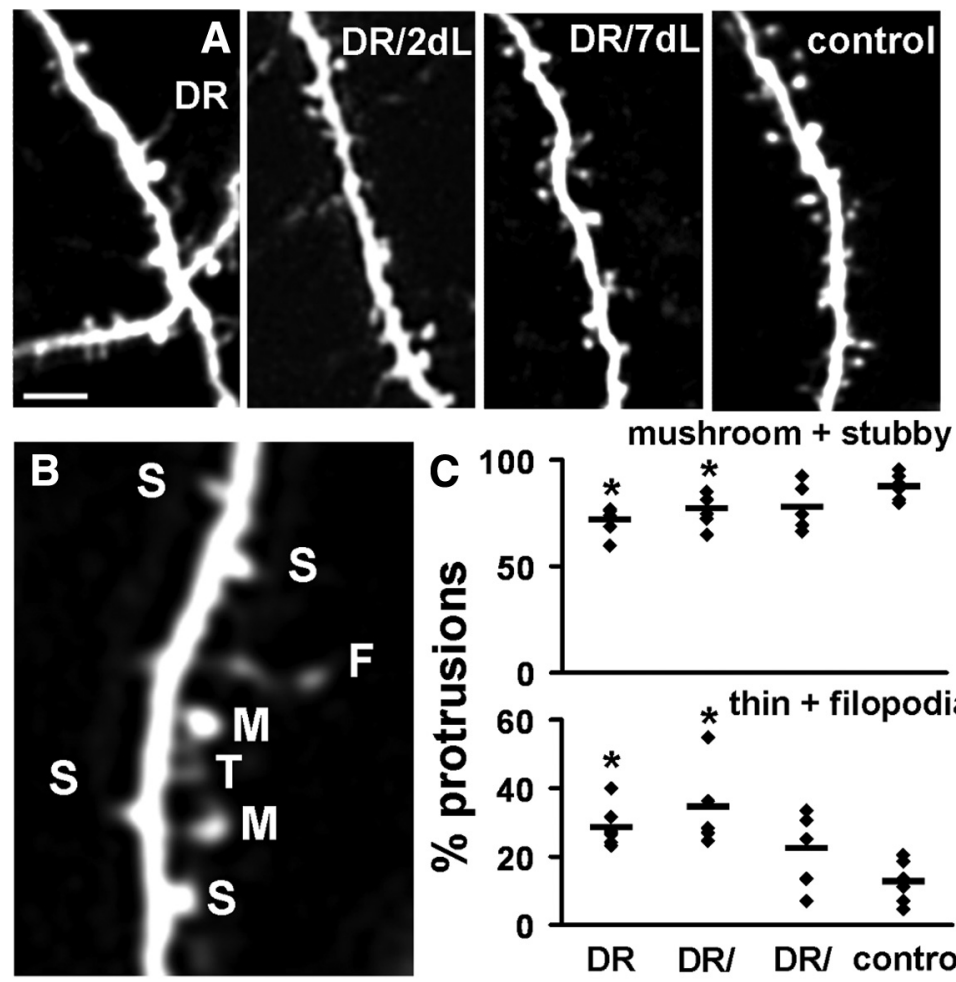
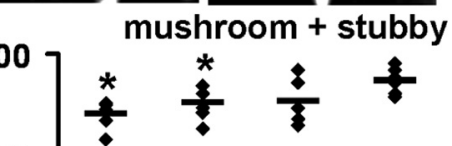

50 1

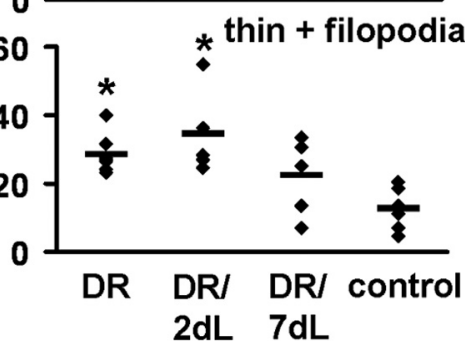

Figure 3. Visual experience affects spine morphology in visual cortex. $\boldsymbol{A}$, High-magnification two-photon images of representative dendrites in the visual cortex of animals reared in different visual environments. Scale bar, $5 \mu \mathrm{m}$. $\boldsymbol{B}$, Image showing the classification of dendritic spines into morphological classes. M, mushroom; S, stubby; T, thin; F, filopodium. C, Variations in the morphologies of dendritic spines across animals with different visual experience. Animals reared in normal conditions show a significantly higher percentage of mushroom and stubby spines compared with animals that have been visually deprived, which show increased numbers of thin spines and filopodia ( ${ }^{*} p<0.05$, comparing control to other conditions). Seven days of reexposure to light after dark-rearing restores the morphological profile of dendritic spines to control levels.

\section{Visual experience affects structural dynamics of dendritic spines}

To determine whether alterations in cortical function had structural correlates at the synaptic level, we used in vivo two-photon time-lapse imaging to visualize layer 5 pyramidal neurons in transgenic mice expressing GFP (Fig. 2). The location of the imaged neurons was confirmed by marking the imaging area using dye injection at the end of the imaging session and identifying V1 in fixed coronal sections (Fig. 2D). We tracked the morphologies of dendritic protrusions in the superficial cortical layers and quantified their structural dynamics over a period of $1-2 \mathrm{~h}$ (Fig. $2 E$ ). At $\mathrm{P} 28$ dendritic protrusions in control mice exhibited mature spine-like morphologies and limited morphological dynamics, as expected from previous studies (Majewska and Sur, 2003; Oray et al., 2004; Majewska et al., 2006). These dynamics were quantified and a motility index was calculated as an absolute average change in base to tip protrusion length over time (for a complete description of measurements in individual animals for all conditions tested, see supplemental Table 1, available at www. jneurosci.org as supplemental material). Filopodia were included in the calculation of overall spine motility index unless stated otherwise. Consistent with previous studies that showed that binocular deprivation leads to increased spine motility during the critical period (Majewska and Sur, 2003), dendritic protrusions in dark-reared animals were more motile than those in controls (DR: $0.029 \pm 0.003 \mu \mathrm{m} / \mathrm{min}, n=287$ protrusions, $n=7$ animals, $7 \pm 2 \%$ filopodia, $0.54 \pm 0.03$ protrusions/ $\mu \mathrm{m}$; control: $0.022 \pm$ $0.001 \mu \mathrm{m} / \mathrm{min}, n=261$ protrusions, $n=6$ animals; $4.3 \pm 2 \%$ filopodia, $0.55 \pm 0.04$ protrusions $/ \mu \mathrm{m}$; $p<0.05$, Fig. $2 F)$. To determine whether light exposure could reverse the effects of dark rearing on spine motility we imaged spine dynamics in animals that were exposed to light. We observed that $2 \mathrm{~d}$ of light exposure did not alter spine motility (DR/2 dl: $0.030 \pm 0.002 \mu \mathrm{m} / \mathrm{min}, n=205$ protrusions, $n=6$ animals, $8 \pm 2 \%$ filopodia, $0.44 \pm 0.05$ protrusions $/ \mu \mathrm{m} ; p=$ 0.63 vs DR; $p<0.05$ vs control), while $7 \mathrm{~d}$ of light exposure reversed the effects of dark rearing returning the motility index to control levels (DR/7 dl: $0.024 \pm 0.002$ $\mu \mathrm{m} / \mathrm{min}, n=176$ protrusions, $n=6$ animals, $7.2 \pm 2 \%$ filopodia, $0.51 \pm 0.05$ protrusions $/ \mu \mathrm{m} ; p=0.59$ vs control; Fig. $2 F)$. It is interesting to note that the timescale of recovery from the effects of dark rearing is similar for spine motility (Fig. $2 F)$ and visually driven cortical activation (Fig. 1D) such that spine motility is high while vision is less able to drive cortical circuits, whereas high visual responsiveness correlates with low spine motility.

\section{Visual experience induces reorganization of dendritic spine morphology and dynamics}

The inverse correlation between visually driven activity in cortical circuits and the dynamics of cortical dendritic spines suggests that visual experience affects cortical activity as well as the organization and maturation of synaptic connectivity and that the structural and functional changes are mutually dependent. Normal development drives generalized changes in dendritic spine morphology and dynamics (Matsuzaki et al., 2001); such developmental changes are prominent in visual cortex, where thin, motile spines bearing weak synapses are replaced by mature stable mushroom-shaped spines with strong synapses embedded within a mature cortical circuit (Harris et al., 1992; Majewska and Sur, 2003; Oray et al., 2006). Thus, we wondered whether visual experience affected the morphology of dendritic spines on layer 5 neurons in visual cortex (Fig. 3). To determine whether dark rearing increased the proportion of immature dendritic spines, we classified spines based on their morphological characteristics (Harris and Kater, 1994). In dark-reared animals, there was a larger proportion of thin spines as well as filopodia, which are thought to be spine precursors (Ziv and Smith, 1996) and are rarely observed in control visual cortex at this age (Majewska and Sur, 2003), along with a decrease in the proportion of mushroom and stubby spine morphologies $(p<0.005$; Fig. $3 A, C)$. Because thin spines and filopodia are generally more dynamic than mushroom and stubby spines (Majewska and Sur, 2003 ) it is possible that the switch in spine subtypes accounts for the increased motility observed in dark-reared animals. In fact no differences in the motility of individual subtypes of spines were observed between control and dark-reared animals $(p>0.05$; supplemental Fig. S2, available at www.jneurosci.org as supplemental material) although all spine types showed similar trends in motility values. This is consistent with previous reports that spine motility and the proportion of thin spines and filopodia 
decrease with enhanced neuronal activity and with development (Dunaevsky et al., 1999; Fischer et al., 2000; Majewska and Sur, 2003; Oray et al., 2006; but see Lendvai et al., 2000), and underscores the effect of dark rearing which both eliminates visually driven activity and delays normal maturation of the visual cortex and the closure of the critical period (Berardi et al., 2003).

We next wondered whether dendritic spine morphology was similarly affected by reexposure to light following dark rearing. Two days of light exposure did not significantly change cortical visually evoked activation (Fig. 1) or the morphological characteristics of dendritic spines $(p>$ 0.05 vs dark reared; $p<0.05$ vs control; Fig. $3 C$ ). Seven days of light exposure following dark rearing, however, shifted the spine population toward more mature morphologies which resembled those in control animals $[p>0.05$ vs control (Fig. $3 C)$ ] similarly to the recovery in visual responsiveness and spine motility.

\section{Inhibitory signaling influences visual responsiveness and dendritic spine characteristics in dark-reared animals} Dark rearing prevents the maturation of inhibitory cells (Tropea et al., 2006) and circuits (Chen et al., 2001; Morales et al., 2002) which are crucial for the proper functioning of the visual cortex (Hensch, 2005). To determine whether enhancing inhibition could restore visual function in dark-reared animals, we treated animals with systemic injections of diazepam in the darkness starting at P21 (DR/7dDIA; Fig. 4). At P28 animals were tested using intrinsic signal and two-photon imaging. Animals treated with diazepam $(n=3)$ in the dark had visually driven cortical activity that was similar to that in control animals ( $p>0.05$ compared with control; Fig. $4 B, D$ ) despite the fact that these animals had never been exposed to visual stimuli before testing. A control animal that had received saline injections in the dark for $7 \mathrm{~d}$ before testing showed poor cortical responsiveness and a poor cortical map similar to dark-reared animals (Fig. 4B, C).

To determine whether diazepam injections resulted in a similar maturation of spine morphology and dynamics in the visual cortex, we examined the motility of dendritic spines in animals treated for $7 \mathrm{~d}$ in the dark with diazepam. Interestingly, dendritic spine motility in diazepam-treated animals was also not significantly different from in control, light-reared animals $(p>0.05$; Fig. $4 F)$. The composition of dendritic spine classes, however, was not reversed by diazepam treatment (Fig. $4 E, G$ ) and was significantly skewed toward more immature phenotypes (thin spines and filopodia) when compared with control animals $(p<$ 0.05 ; Fig. $4 G$ ). These results suggest that diazepam treatment can partially rescue the effects of light deprivation.

\section{Brief light exposure following dark rearing elicits rapid changes in cortical organization}

At $2 \mathrm{~d}$ of light exposure following dark rearing we observed no changes in the ability of light to drive cortical activity or in den- dritic spines dynamics. However, previous studies have shown rapid molecular changes in visual cortex following very brief $(\sim 2$ h) exposure to light (Tropea et al., 2001; Cotrufo et al., 2003). We decided, therefore, to examine whether cortical map and dendritic spine organization also changed rapidly following light exposure. We exposed an additional set of mice which were darkreared from birth till P28 to $2 \mathrm{~h}$ of light (Fig. $5 A$; DR/2hL; $n=4$ ). Surprisingly, we found that $2 \mathrm{~h}$ of light exposure after dark rearing resulted in a significant increase in cortical responsiveness to visual stimuli and the early emergence of a cortical visual map (Fig. $5 B, C$ ). Visually driven cortical activation was significantly elevated when compared with both dark-reared (DR) and $2 \mathrm{~d}$ light-exposed $(\mathrm{DR} / 2 \mathrm{dl})$ animals $(p<0.05)$. To determine the timeline of cortical changes for light exposure we exposed additional animals to different durations of light after dark rearing before assaying visual function using intrinsic signal imaging (supplemental Fig. S3, available at www.jneurosci.org as supplemental material). Visual responsiveness peaked at $4 \mathrm{~h}$ of light exposure and returned to the level of that in dark-reared animals by $2 \mathrm{~d}$ of exposure. This suggests that light exposure very rapidly triggers the activation of cortical circuits leading to recovery of visual responses. This early recovery, however, is not maintained, suggesting that two phases of reorganization underlie recovery from dark-rearing: (a) a rapid, transient change that potentiates activity of visual pathways and that reorganizes cortical networks, followed by (b) a slow recovery of cortical responsiveness. We 


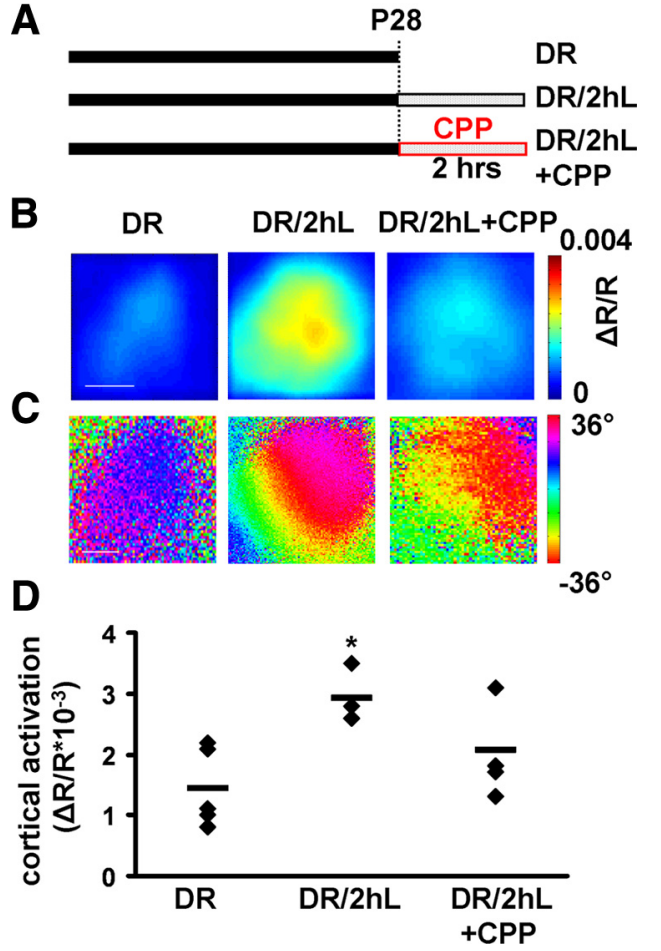

Figure 5. Light exposure induces rapid functional reorganization mediated by NMDA receptors. $\boldsymbol{A}$, Schematic of experimental timeline. Three groups of animals were used: animals reared in the darkness from birth (DR; same as Fig. 1,3,4), DR animals exposed to light for $2 \mathrm{~h}$ before imaging (DR/2hL) and DR animals in which the NMDA antagonist CPP was injected systemically $30 \mathrm{~min}$ before $2 \mathrm{~h}$ of light exposure (DR/2hL + CPP). $\boldsymbol{B}$, Representative images of the cortical intrinsic signal in response to light in individual mice from the different groups imaged. Red hues indicate strong activation, as per $d R / R$ scale at right. Mice exposed to light for $2 \mathrm{~h}$ show strong visually driven responses similar to control mice. Mice pretreated with the NMDA receptor antagonist CPP do not show increased visual responses after brief light exposure. Scale bar, $0.5 \mathrm{~mm}$. C, Representative retinotopic maps of elevation in individual mice, as per key at right. $\boldsymbol{D}$, Quantification of the amplitude of visually evoked cortical activation in the three groups of animals. Brief light exposure significantly increased visually evoked cortical activation ${ }^{*} p<0.05$ compared with dark-reared animals). This effect was prevented by systemic injection of (PP before light exposure.

provide evidence below that the early rapid phase is dependent on NMDA receptor signaling.

\section{Brief light exposure elicits rapid changes at the level of dendritic spines}

To determine whether synaptic changes were responsible for the rapid changes in cortical function observed after $2 \mathrm{~h}$ of light exposure, we examined the motility of dendritic spines in the visual cortex of these animals. Because our previous results suggested that visual responsiveness was inversely related to spine motility and given that spine motility is dampened by synaptic glutamate receptor signaling (Fischer et al., 2000; Korkotian and Segal, 2001; Oray et al., 2006) we reasoned that a short period of visual experience would reverse the effects of dark rearing on spine dynamics. Surprisingly, we found that $2 \mathrm{~h}$ of light exposure significantly upregulated dendritic spine dynamics $(0.036 \pm 0.004 \mu \mathrm{m} / \mathrm{min} n=285$ protrusions, $n=7$ animals, $9.9 \pm 1.8 \%$ filopodia, $0.56 \pm 0.07$ protrusions $/ \mu \mathrm{m}$ ) compared with control $(p<0.01)$ and dark rearing conditions $(p<0.05$; Fig. $6 A-C)$. Furthermore, increased spine motility was accompanied by increased expression of immature protrusions (thin spines and filopodia) and a decreased incidence of mushroom and stubby spines ( $p<0.05$ vs DR; Fig. $6 D, E)$. This suggests that rapid effects of light exposure are mediated by immature protrusions which are more motile than in DR and control animals. In addition, the findings indicate that the sudden activation of circuitry by visual stimuli induces a transient, destabilizing phase where a functional increase in cortical responsiveness is paired with a rapid remodeling of glutamatergic connections. These data are consistent with other studies which reported that the immediate effect of activating sensory experience was to increase glutamatergic transmission (Lu and Constantine-Paton, 2004) and spine dynamics (Hofer et al., 2009).

To determine whether the dynamic changes observed in dendritic spines following brief light exposure were the result of new protrusion outgrowth or a destabilization of existing spines, we imaged the same animals chronically before and after light exposure (Fig. 7). We found that $2 \mathrm{~h}$ of light exposure induced a significant outgrowth of dendritic protrusions (formation: $20 \pm$ $3.6 \%$ in light-exposed animals (DR_LT), $n=5$, vs $4.2 \pm 1.1 \%$ in animals maintained in the dark (DR_DR), $n=5$; vs $6.7 \pm 1.3 \%$ in light-reared control (LT_LT), $n=3 ; p<0.05$ compared with both DR_DR and LT_LT) without affecting elimination (elimination: $5.4 \pm 2.1 \%$ DR_LT; $9.4 \pm 4.8 \%$, DR_DR, $5.7 \pm 2.3 \%$ LT_LT $p>0.05$; Fig. $7 B$ ). Half of these new protrusions (49\%) had immature morphologies and were classified as thin spines or filopodia.

To understand whether the transient nature of this response to light exposure was mediated by rapid changes in synaptic architecture, we also imaged mice before and after $2 \mathrm{~d}$ of exposure to light. Interestingly the rates of protrusion formation were matched in both dark-reared animals exposed to light and control animals reared in a normal light environment (formation: $10.1 \pm 2.3 \%$, DR_LT, $n=4 ; 12.3 \pm 1.7 \%$, LT_LT, $n=4 ; p>$ 0.05 ; Fig. $7 C$ ). These rates were approximately half of those observed in animals exposed to light for only $2 \mathrm{~h}$ suggesting that initial outgrowth of new protrusions is not maintained with longer light exposure times. Interestingly the rates of protrusion elimination were significantly higher in dark-reared lightexposed animals (elimination: $19.2 \pm 1.9 \%$, DR_LT, $n=4 ; 7.9 \pm$ $4 \%$, LT_LT, $n=4 ; p<0.05$; Fig. $7 C$ ) suggesting that longer term light exposure leads to the elimination of both newly generated and preexisting protrusions.

We verified these results in two animals in which we followed the same dendritic sections at three time points: before light exposure, $2 \mathrm{~h}$ after light exposure and $2 \mathrm{~d}$ after light exposure (Fig. $7 A)$. Again we found pronounced outgrowth of new, immature protrusions after $2 \mathrm{~h}$ of light exposure (formation: $22 \pm 5 \%$ ), but many of these were not maintained after $2 \mathrm{~d}$ in the light. We computed a survival fraction and found that $30 \pm 4 \%$ of new protrusions were maintained after $2 \mathrm{~d}$ of light exposure, matching the value obtained in control light-reared animals $(33 \% ; n=$ 2 ). The elimination of preexisting spines, however, was almost doubled in dark-reared light-exposed animals $(21.8 \pm 3 \%)$ when compared with light-reared controls $(13.8 \pm 1.2 \%)$, again suggesting that normal sensory experience is required to maintain neural connectivity.

\section{Rapid changes in cortical organization elicited by light are mediated by NMDA receptor signaling}

We were interested in determining the molecular mechanisms responsible for the fast remodeling phase in which cortical responsiveness to visual stimuli and map organization is rapidly restored by vision. Since exposure to light is likely to trigger in- 


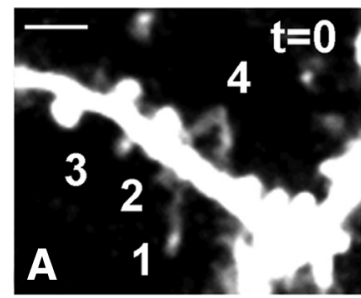

B
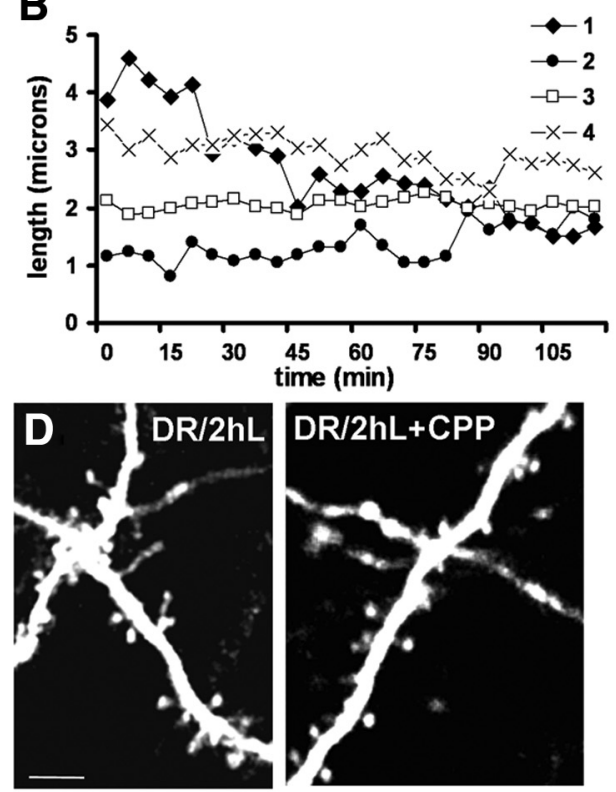
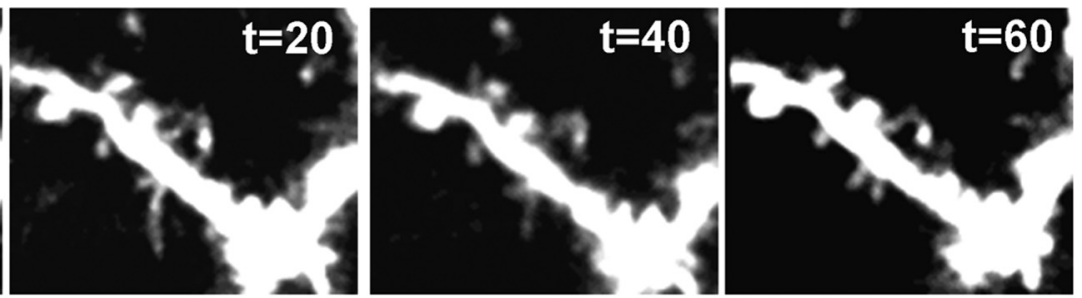

C
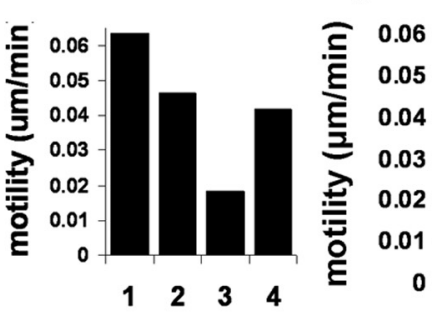

E

mushroom + stubby

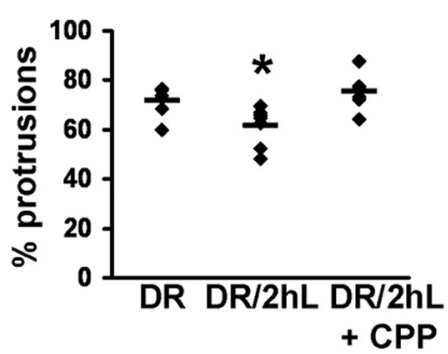

*

$\bullet$
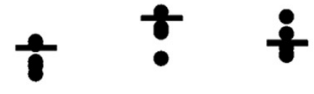

DR DR/2hL DR/2hL

+ CPP

thin + filopodia

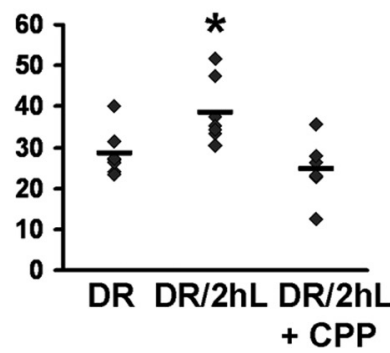

Figure 6. Light exposure induces rapid structural reorganization mediated by NMDA receptors. $A$, Time-lapse image of dendritic spines in the visual cortex of a dark-reared mouse in vivo following light exposure for $2 \mathrm{~h}$ at P28. Images shown were taken 20 min apart. Scale bar, $2 \mu \mathrm{m}$. Dendritic protrusions are highly motile in this group of animals (DR/2hL). $\boldsymbol{B}$, Lengths of the $4 \mathrm{protrusions}$ labeled in $\mathrm{A}$ are shown in the left panel plotted over $2 \mathrm{~h}$. Notice the large changes observed in length over this timescale. The right panel shows the motility index for the same four protrusions. Protrusion 1 was classified as a filopodium due to its length. Not all spines are highly motile. Spine 3 exhibits length changes and a motility index typical of spines in control mice at this age. C, Brief light exposure rapidly increases spine motility in dark-reared animals $\left({ }^{*} p<0.05\right)$. This effect is prevented when CPP is administered systemically before light exposure. D, High-magnification two-photon images of representative dendrites in the visual cortex of animals exposed to light for $2 \mathrm{~h}$ following dark rearing. Notice the increased numbers of thin spines and filopodia in dark-reared animals briefly exposed to light. Animals pretreated with (PP before light exposure have fewer thin spines and filopodia than untreated animals, but similar in proportion to dark-reared animals. Scale bar, $5 \mu \mathrm{m}$. $E$, Brief light exposure increases the proportion of thin protrusions (thin spines and filopodia) and decreases the proportion of mushroom and stubby spines compared with DR animals $\left({ }^{*} p<0.05\right)$. The reorganization of dendritic spine morphology is prevented by administration of CPP before light exposure.

creased glutamatergic activity in cortical circuits, we focused on NMDA receptor signaling. NMDA receptors are a likely mediator of rapid light-induced changes as their expression is regulated by dark rearing and brief light exposure, and NMDA receptors have been implicated in cortical rearrangements and many different forms of synaptic plasticity (Philpot et al., 2001). To determine whether NMDA receptors mediate the rapid effects of light exposure following dark rearing we investigated visually driven cortical activity and dendritic spine motility in animals that were injected systemically with CPP, a blocker of NMDA receptors, before being exposed to light for $2 \mathrm{~h}$ (DR/2hLCPP; $n=3$ animals; Fig. 5A). CPP administration prevented the increase in visually driven cortical activation and diminished the precision of the visual map ( $p>0.05$ vs DR; Fig. $5 B-E$ ). To ensure that CPP did not affect visual responsiveness directly, we administered CPP to light-reared animals $(n=3)$ and imaged using the same timeline as the DR/2hLCPP animals. CPP administration did not affect visually evoked responses or map organization in light-reared animals $(p>0.05)$.

CPP administration also decreased dendritic spine motility and prevented the shift in the spine population toward more immature phenotypes ( $p>0.05$ vs DR; Fig. $6 C-E$ ). To ensure that CPP administration did not affect spine morphology and dynamics we administered CPP to animals without light exposure $(n=3)$; this did not change spine motility levels $(0.028 \pm$ $0.001 \mu \mathrm{m} / \mathrm{min}, n=98$ spines; 3 animals) or spine morphology relative to dark-reared animals $(p>0.05)$. Together these results suggest that many of the early effects of light exposure on cortical organization and drive are effected by NMDA receptor-mediated signaling.

\section{Discussion}

Activity-driven changes in brain circuitry have both functional and structural components (Rittenhouse and Majewska, 2009). To determine the relationship between structural changes at single synapses and functional changes in cortical networks in vivo, we imaged dendritic spines on layer 5 neurons and network activity in the visual cortex while manipulating cortical activity levels by dark-rearing mice and reexposing them to light. Over time periods of days, we found an inverse correspondence between dendritic spine structural dynamics and visually evoked cortical function. Dark-reared mice showed significant upregulation of dendritic spine motility and disrupted visual processing. Light exposure for up to $7 \mathrm{~d}$ following dark-rearing led to a slow increase in visually evoked cortical processing as well as a stabilization of dendritic spine structure that could be partially mim- 

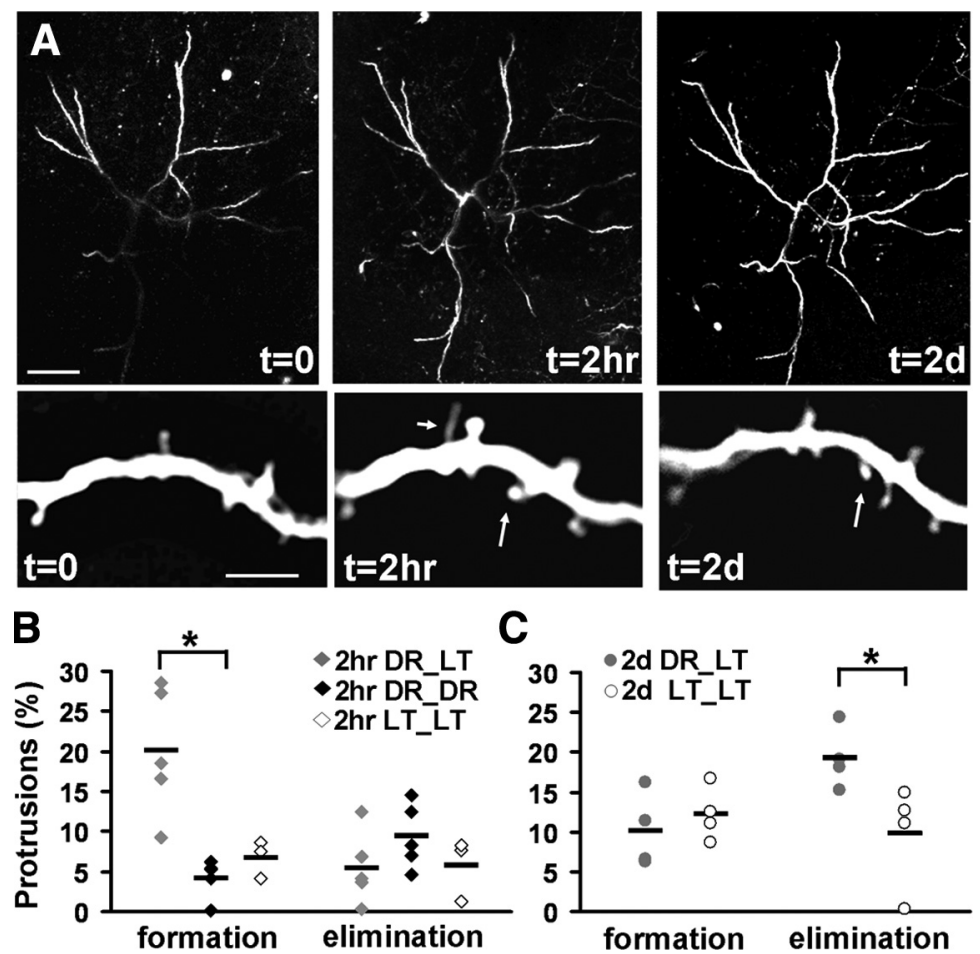

Figure 7. Light exposure induces a rapid, transient outgrowth of dendritic protrusions. $A$, Top, A dendritic arbor imaged over $2 \mathrm{~d}$ in a dark-reared animal exposed to light after the first imaging session. Bottom, A higher magnification of a dendrite bearing dendritic spines. Notice the outgrowth of a filopodium (arrowhead) and spine (arrow). The filopodium withdraws after $2 \mathrm{~d}$ of light exposure but the spine is maintained. Scale bar, $30 \mu \mathrm{m}$ (top); $5 \mu \mathrm{m}$ (bottom). $\boldsymbol{B}$, The formation of dendritic protrusions is enhanced after $2 \mathrm{~h}$ of light exposure in dark-reared animals relative to animals maintained in the dark and relative to normal light-reared controls ( ${ }^{*} p<0.05$ ), while the rate of elimination is not altered. $\boldsymbol{C}$, The percentage of new protrusions formed between the end of the dark rearing period and after $2 \mathrm{~d}$ of light exposure is not different from that over a $2 \mathrm{~d}$ period in light-reared animals. However, the elimination of spines is significantly enhanced suggesting a pruning of preexisting synapses.

icked by enhancing inhibition in the absence of light exposure. Interestingly, very brief $(2 \mathrm{~h}$ ) periods of light exposure led to an NMDA-dependent rapid reorganization of cortical networks with an early emergence of visually evoked cortical activation and enhanced spine dynamics. These results suggest that structural and functional synaptic changes in vivo are linked, but can undergo complex transient changes when perturbed before their relationship is reestablished.

The effect of vision on structure and function in visual cortex Total absence of visual experience (dark-rearing) delays the normal maturation of cortical neurons properties (Fagiolini et al., 1994) and the molecular events which occur at eye opening ( $\mathrm{Lu}$ and Constantine-Paton, 2004) bear a striking resemblance to those that occur during light reexposure after dark-rearing (Quinlan et al., 1999; Cotrufo et al., 2003). Light exposure following dark-rearing facilitates the recovery of functional properties (Buisseret et al., 1982; Mower et al., 1983), suggesting that normal developmental processes are restored once visual deprivation is discontinued. These observations make dark rearing an intriguing model for studying experience-dependent development and the molecules involved in circuitry maturation (Ciucci et al., 2007). We used this model for investigating the temporal role of activity in the structural and functional reorganization of cortical circuitry.

Following dark rearing, visual responses were weak and dendritic spines maintained immature, unstable morphologies, suggesting that weak, inappropriate synapses lead to dysfunctional cortical circuitry. This agrees with a role of dark rearing in delay- ing developmental processes because dendritic spine morphology (including the emergence of filopodia) (Ziv and Smith, 1996) and spine motility are developmentally regulated (Dunaevsky et al., 1999).

Surprisingly, we observed two distinct phases of cortical recovery after light reexposure: one transient, occurring very rapidly ( $\sim 2 \mathrm{~h})$, and one gradual occurring over a period of days. Initially, very rapid changes in cortical activation were accompanied by an outgrowth of new protrusions and increased spine motility mediated by highly motile thin spines and filopodia. Thus, the first stage of lightinduced recovery is dependent on immature protrusions suggesting it results from the rapid growth and formation of new synapses.

After $2 \mathrm{~d}$ of light exposure, cortical tuning returned to dark-rearing levels and the proportion of thin spines and filopodia decreased. Spine motility was not significantly different from dark-rearing levels. It is puzzling that the large effects of light on cortical function observed after $2 \mathrm{~h}$ are reversed after longer light exposure. Our chronic imaging experiments suggest that the large scale formation of new synapses at early time points cannot be maintained and few are stabilized. This appears to be compounded by an increased loss of preexisting protrusions which may signal a start to the lightmediated pruning of inappropriate synapses made in the dark and establishment of connections that underlie the visual map. Interestingly, these results agree with a previous report that showed that 2-4 d of light exposure was not sufficient to close the critical period in dark-reared mice (Iwai et al., 2003), suggesting that recovery from dark-rearing in these animals requires longer light exposures. Likewise, previous work in the somatosensory system showed that recovery from deprivation accelerates developmental spine elimination (Grutzendler et al., 2002; Zuo et al., 2005). After 7 d of light exposure spine motility and visual function approached control levels, suggesting that longer periods of light exposure may allow full recovery of structural and functional characteristics, although some studies have also reported a lack of recovery in dendritic structure after longer periods of reexposure (Wallace and Bear, 2004).

Our results show that the slow time course (over days), for light-mediated structural and functional changes in visual cortex, are similar. This is particularly evident in the correlation between the spine motility and visual responsiveness $\left(r^{2}=0.873\right)$ when comparing dark-reared, 2 and $7 \mathrm{~d}$ light-exposed and control animals. Interestingly, the $2 \mathrm{~h}$ time point breaks this trend (and is excluded from the analysis above) while blocking NMDA activity with CPP restores the direct correlation between spine motility and cortical activation.

\section{Spine motility and synaptic function}

The phenomenon of spine motility is not well characterized but has been hypothesized to correlate with changes in synaptic connectivity (Bonhoeffer and Yuste, 2002). Spine motility is highest 
during synaptogenesis (Ziv and Smith, 1996; Dunaevsky et al., 1999; Lendvai et al., 2000; Oray et al., 2006), where it may promote synapse formation through an active postsynaptic search mechanism. Spine motility in more adult animals, such as in this study, may be indicative of a switch in presynaptic partners: a weak synapse may explore the extracellular space for a new presynaptic partner; it may signal fluctuations in synaptic strength (Matsuzaki et al., 2004; Zhou et al., 2004); or it might be a byproduct of other changes effected by manipulations of vision, e.g., alterations affecting the dendritic actin or extracellular matrix structure. In this last case spine motility would serve as a readout of molecular changes tied to synaptic plasticity. In our experiments increased motility corresponded to a period of high spine turnover. Therefore we believe it more likely that spine motility indicates an outgrowth or retraction of protrusions to alter network connectivity. It is important to note that our measure of spine motility, while robust, does not capture many aspects of dynamic spine morphology which may have different cellular functions and may be regulated independently of one another.

\section{Mechanisms of structural and functional plasticity in the visual system}

Our results suggest that NMDA-receptor signaling is crucial for the initiation of the first, rapid phase of light-mediated recovery from dark-rearing when new protrusions are rapidly created. NMDA receptors modulate dendritic spine motility (Fischer et al., 2000), synapse strength and dendritic spine morphology (Matsuzaki et al., 2004; Zhou et al., 2004) as well as filopodia extension (Maletic-Savatic et al., 1999) and spinogenesis (Engert and Bonhoeffer, 1999). NMDA receptors mediate outgrowth of dendritic arbors in response to light exposure in the optic tectum of tadpoles through Rho-GTPases which can induce cytoskeletal changes (Sin et al., 2002), suggesting that a similar mechanism may be responsible for remodeling dendritic spines in the mammalian brain. Interestingly, the activity-dependent switch in NMDA receptor composition happens within $2 \mathrm{~h}$ from light exposure after dark-rearing (Quinlan et al., 1999; Philpot et al., 2001). Thus NMDA-receptor dependent potentiation appears to be responsible for the first rapid phase of recovery and the rapid switch in NMDA receptor subunits mediated by light may then terminate this phase and underlie a reversal in dendritic spine motility and cortical visual function. Additionally, NMDA receptor blockade has been shown to disrupt the enhancement of spine elimination following recovery from sensory deprivation (Zuo et al., 2005) suggesting that NMDA-mediated signaling could also play a prominent role in the increased pruning of spines within $2 \mathrm{~d}$ of light reexposure after dark rearing.

Slow changes elicited by light after dark-rearing may rely on homeostatic mechanisms that engage excitation, inhibition and neuronal excitability. Inhibition appears as an attractive mediator of slow changes because of its importance in regulating the plasticity and maturity of visual cortical circuits (Hensch, 2005). Our work suggests that increasing inhibition improves visual responsiveness even in the absence of light. It does not, however, restore structural maturation of excitatory connectivity and dendritic spines maintain immature morphologies suggesting that inhibitory mechanisms are only part of the story. An intriguing possibility is that homeostatic mechanisms regulate both excitatory and inhibitory function in tandem (Kanold et al., 2009). Thus dark rearing reduces both excitatory and inhibitory signaling (Tropea et al., 2006), while light exposure reactivates both systems in an interactive activity-dependent manner.

\section{Conclusions}

In summary, our experiments demonstrate a high degree of correlation between structural dynamics of synapses and functional activation of the visual cortex during manipulations of visual activity. We show that the effects of total lack of experience are reversible, with correlated structural and functional changes on slower timescales. Light exposure acts in a biphasic manner, however, whereby a rapid, transient, reactivation of visual circuitry mediated by NMDA receptors is followed by a slow stabilization phase that may rely on homeostatic mechanisms which match inhibitory and excitatory systems and refine visually responsive circuitry. These findings likely apply generally to processes that drive the maturation and experience-dependent development of cortical circuitry.

\section{References}

Berardi N, Pizzorusso T, Ratto GM, Maffei L (2003) Molecular basis of plasticity in the visual cortex. Trends Neurosci 26:369-378.

Bonhoeffer T, Yuste R (2002) Spine motility. Phenomenology, mechanisms, and function. Neuron 35:1019-1027.

Brakeman PR, Lanahan AA, O’Brien R, Roche K, Barnes CA, Huganir RL, Worley PF (1997) Homer: a protein that selectively binds metabotropic glutamate receptors. Nature 386:284-288.

Buisseret P, Gary-Bobo E, Imbert M (1982) Plasticity in the kitten's visual cortex: effects of the suppression of visual experience upon the orientational properties of visual cortical cells. Brain Res 256:417-426.

Chen L, Yang C, Mower GD (2001) Developmental changes in the expression of $\operatorname{GABA}(\mathrm{A})$ receptor subunits (alpha(1), alpha(2), alpha(3)) in the cat visual cortex and the effects of dark rearing. Brain Res Mol Brain Res 88:135-143.

Ciucci F, Putignano E, Baroncelli L, Landi S, Berardi N, Maffei L (2007) Insulin-like growth factor 1 (IGF-1) mediates the effects of enriched environment (EE) on visual cortical development. PLoS One 2:e475.

Cotrufo T, Viegi A, Berardi N, Bozzi Y, Mascia L, Maffei L (2003) Effects of neurotrophins on synaptic protein expression in the visual cortex of darkreared rats. J Neurosci 23:3566-3571.

Czepita D, Reid SN, Daw NW (1994) Effect of longer periods of dark rearing on NMDA receptors in cat visual cortex. J Neurophysiol 72:1220-1226.

Dunaevsky A, Tashiro A, Majewska A, Mason C, Yuste R (1999) Developmental regulation of spine motility in the mammalian central nervous system. Proc Natl Acad Sci U S A 96:13438-13443.

Engert F, Bonhoeffer T (1999) Dendritic spine changes associated with hippocampal long-term synaptic plasticity. Nature 399:66-70.

Fagiolini M, Pizzorusso T, Berardi N, Domenici L, Maffei L (1994) Functional postnatal development of the rat primary visual cortex and the role of visual experience: dark rearing and monocular deprivation. Vision Res 34:709-720.

Feng G, Mellor RH, Bernstein M, Keller-Peck C, Nguyen QT, Wallace M, Nerbonne JM, Lichtman JW, Sanes JR (2000) Imaging neuronal subsets in transgenic mice expressing multiple spectral variants of GFP. Neuron 28:41-51.

Fischer M, Kaech S, Wagner U, Brinkhaus H, Matus A (2000) Glutamate receptors regulate actin-based plasticity in dendritic spines. Nat Neurosci 3:887-894.

Franklin KBJ, Paxinos G (1997) The mouse brain in stereotaxic coordinates. San Diego: Academic.

Frenkel MY, Bear MF (2004) How monocular deprivation shifts ocular dominance in visual cortex of young mice. Neuron 44:917-923.

Gordon JA, Stryker MP (1996) Experience-dependent plasticity of binocular responses in the primary visual cortex of the mouse. J Neurosci 16:3274-3286

Grutzendler J, Kasthuri N, Gan WB (2002) Long-term dendritic spine stability in the adult cortex. Nature 420:812-816.

Harris KM, Kater SB (1994) Dendritic spines: cellular specializations imparting both stability and flexibility to synaptic function. Annu Rev Neurosci 17:341-371

Harris KM, Jensen FE, Tsao B (1992) Three-dimensional structure of dendritic spines and synapses in rat hippocampus (CA1) at postnatal day 15 and adult ages: implications for the maturation of synaptic physiology and long-term potentiation. J Neurosci 12:2685-2705. 
Hensch TK (2005) Critical period mechanisms in developing visual cortex. Curr Top Dev Biol 69:215-237.

Hofer SB, Mrsic-Flogel TD, Bonhoeffer T, Hübener M (2009) Experience leaves a lasting structural trace in cortical circuits. Nature 457:313-317.

Holtmaat A, Wilbrecht L, Knott GW, Welker E, Svoboda K (2006) Experience-dependent and cell-type-specific spine growth in the neocortex. Nature 441:979-983.

Iwai Y, Fagiolini M, Obata K, Hensch TK (2003) Rapid critical period induction by tonic inhibition in visual cortex. J Neurosci 23:6695-6702.

Kanold PO, Kim YA, GrandPre T, Shatz CJ (2009) Co-regulation of ocular dominance plasticity and NMDA receptor subunit expression in glutamic acid decarboxylase-65 knock-out mice. J Physiol 587:2857-2867.

Korkotian E, Segal M (2001) Regulation of dendritic spine motility in cultured hippocampal neurons. J Neurosci 21:6115-6124.

Lander C, Kind P, Maleski M, Hockfield S (1997) A family of activitydependent neuronal cell-surface chondroitin sulfate proteoglycans in cat visual cortex. J Neurosci 17:1928-1939.

Lendvai B, Stern EA, Chen B, Svoboda K (2000) Experience-dependent plasticity of dendritic spines in the developing rat barrel cortex in vivo. Nature 404:876-881.

Li Y, Fitzpatrick D, White LE (2006) The development of direction selectivity in ferret visual cortex requires early visual experience. Nat Neurosci 9:676-681.

Lu W, Constantine-Paton M (2004) Eye opening rapidly induces synaptic potentiation and refinement. Neuron 43:237-249.

Majewska A, Sur M (2003) Motility of dendritic spines in visual cortex in vivo: changes during the critical period and effects of visual deprivation. Proc Natl Acad Sci U S A 100:16024-16029.

Majewska AK, Sur M (2006) Plasticity and specificity of cortical processing networks. Trends Neurosci 29:323-329.

Majewska AK, Newton JR, Sur M (2006) Remodeling of synaptic structure in sensory cortical areas in vivo. J Neurosci 26:3021-3029.

Majewska A, Yiu G, Yuste R (2000) A custom-made two-photon microscope and deconvolution system. Pflugers Arch 441:398-408.

Maletic-Savatic M, Malinow R, Svoboda K (1999) Rapid dendritic morphogenesis in CA1 hippocampal dendrites induced by synaptic activity. Science 283:1923-1927.

Mataga N, Mizuguchi Y, Hensch TK (2004) Experience-dependent pruning of dendritic spines in visual cortex by tissue plasminogen activator. Neuron 44:1031-1041.

Matsuzaki M, Ellis-Davies GC, Nemoto T, Miyashita Y, Iino M, Kasai H (2001) Dendritic spine geometry is critical for AMPA receptor expression in hippocampal CA1 pyramidal neurons. Nat Neurosci 4:10861092.

Matsuzaki M, Honkura N, Ellis-Davies GC, Kasai H (2004) Structural basis of long-term potentiation in single dendritic spines. Nature 429:761-766.

Morales B, Choi SY, Kirkwood A (2002) Dark rearing alters the development of GABAergic transmission in visual cortex. J Neurosci 22:80848090 .

Mower GD, Christen WG, Caplan CJ (1983) Very brief visual experience eliminates plasticity in the cat visual cortex. Science 221:178-180.

Noguchi J, Matsuzaki M, Ellis-Davies GC, Kasai H (2005) Spine-neck ge- ometry determines NMDA receptor-dependent calcium signaling in dendrites. Neuron 46:609-622.

Oray S, Majewska A, Sur M (2004) Dendritic spine dynamics are regulated by monocular deprivation and extracellular matrix degradation. Neuron 44:1021-1030.

Oray S, Majewska A, Sur M (2006) Effects of synaptic activity on dendritic spine motility of developing cortical layer v pyramidal neurons. Cereb Cortex 16:730-741.

Philpot BD, Sekhar AK, Shouval HZ, Bear MF (2001) Visual experience and deprivation bidirectionally modify the composition and function of NMDA receptors in visual cortex. Neuron 29:157-169.

Quinlan EM, Philpot BD, Huganir RL, Bear MF (1999) Rapid, experiencedependent expression of synaptic NMDA receptors in visual cortex in vivo. Nat Neurosci 2:352-357.

Ramón y Cajal S (1904) In: La textura del sistema nerviosa del hombre y los vertebrados. Madrid: Moya.

Rittenhouse CD, Majewska AK (2009) Synaptic mechanisms of activitydependent remodeling in visual cortex. J Exp Neurosci 2:23-41.

Sherman SM, Spear PD (1982) Organization of visual pathways in normal and visually deprived cats. Physiol Rev 62:738-855.

Sin WC, Haas K, Ruthazer ES, Cline HT (2002) Dendrite growth increased by visual activity requires NMDA receptor and Rho GTPases. Nature 419:475-480.

Smith SL, Trachtenberg JT (2007) Experience-dependent binocular competition in the visual cortex begins at eye opening. Nat Neurosci 10:370-375.

Tropea D, Capsoni S, Tongiorgi E, Giannotta S, Cattaneo A, Domenici L (2001) Mismatch between BDNF mRNA and protein expression in the developing visual cortex: the role of visual experience. Eur J Neurosci 13:709-721.

Tropea D, Kreiman G, Lyckman A, Mukherjee S, Yu H, Horng S, Sur M (2006) Gene expression changes and molecular pathways mediating activity-dependent plasticity in visual cortex. Nat Neurosci 9:660-668.

Valverde F (1971) Rate and extent of recovery from dark rearing in the visual cortex of the mouse. Brain Res 33:1-11.

Wallace W, Bear MF (2004) A morphological correlate of synaptic scaling in visual cortex. J Neurosci 24:6928-6938.

Winkelmann E, Brauer K, Werner L (1976) [Studies on the spine density in lamina $\mathrm{V}$ pyramidal cells of the visual cortex in young and subadult rats after dark-rearing and destruction of the dorsal nucleus in the lateral geniculate body]. J Hirnforsch 17:489-500.

Yang CB, Zheng YT, Li GY, Mower GD (2002) Identification of Munc13-3 as a candidate gene for critical-period neuroplasticity in visual cortex. J Neurosci 22:8614-8618.

Zhou Q, Homma KJ, Poo MM (2004) Shrinkage of dendritic spines associated with long-term depression of hippocampal synapses. Neuron 44:749-757.

Ziv NE, Smith SJ (1996) Evidence for a role of dendritic filopodia in synaptogenesis and spine formation. Neuron 17:91-102.

Zuo Y, Yang G, Kwon E, Gan WB (2005) Long-term sensory deprivation prevents dendritic spine loss in primary somatosensory cortex. Nature 436:261-265. 